\title{
Planar Diameter via Metric Compression
}

\author{
Jason Li \\ jmli@cs.cmu.edu \\ CMU \\ United States
}

\author{
Merav Parter \\ merav.parter@weizmann.ac.il \\ Weizmann Institute \\ Israel
}

\begin{abstract}
We develop a new approach for distributed distance computation in planar graphs that is based on a variant of the metric compression problem recently introduced by Abboud et al. [SODA'18]. In our variant of the Planar Graph Metric Compression Problem, one is given an $n$-vertex planar graph $G=(V, E)$, a set of $S \subseteq V$ source terminals lying on a single face, and a subset of target terminals $T \subseteq V$. The goal is to compactly encode the $S \times T$ distances.

One of our key technical contributions is in providing a compression scheme that encodes all $S \times T$ distances using $\widetilde{O}(|S| \cdot \operatorname{poly}(D)+$ $|T|)$ bits, for unweighted graphs with diameter $D$. This significantly improves the state of the art of $\widetilde{O}\left(|S| \cdot 2^{D}+|T| \cdot D\right)$ bits. We also consider an approximate version of the problem for weighted graphs, where the goal is to encode $(1+\epsilon)$ approximation of the $S \times T$ distances, for a given input parameter $\epsilon \in(0,1]$. Here, our compression scheme uses $\widetilde{O}($ poly $(|S| / \epsilon)+|T|)$ bits. In addition, we describe how these compression schemes can be computed in near-linear time. At the heart of this compact compression scheme lies a VCdimension type argument on planar graphs, using the well-known SauerâĂŹ's lemma.

This efficient compression scheme leads to several improvements and simplifications in the setting of diameter computation, most notably in the distributed setting:

- There is an $\widetilde{O}\left(D^{5}\right)$-round randomized distributed algorithm for computing the diameter in planar graphs, w.h.p.

- There is an $\widetilde{O}\left(D^{3}\right)+D^{2}$ poly $(\log n / \epsilon)$-round randomized distributed algorithm for computing a $(1+\epsilon)$ approximation for the diameter in weighted planar graphs, with unweighted diameter $D$, w.h.p.
\end{abstract}

No sublinear round algorithms were known for these problems before. These distributed constructions are based on a new recursive graph decomposition that preserves the (unweighted) diameter of each of the subgraphs up to a logarithmic term. Using this decomposition, we also get an exact SSSP tree computation within $\widetilde{O}\left(D^{2}\right)$ rounds.

\section{CCS CONCEPTS}

- Mathematics of computing $\rightarrow$ Graph algorithms; • Theory of computation $\rightarrow$ Shortest paths; Distributed algorithms.

Permission to make digital or hard copies of all or part of this work for personal or classroom use is granted without fee provided that copies are not made or distributed for profit or commercial advantage and that copies bear this notice and the full citation on the first page. Copyrights for components of this work owned by others than ACM must be honored. Abstracting with credit is permitted. To copy otherwise, or republish, to post on servers or to redistribute to lists, requires prior specific permission and/or a fee. Request permissions from permissions@acm.org.

STOC '19, fune 23-26, 2019, Phoenix, AZ, USA

(c) 2019 Association for Computing Machinery.

ACM ISBN 978-1-4503-6705-9/19/06 . .\$15.00

https://doi.org/10.1145/3313276.3316358

\section{KEYWORDS}

diameter, planar graphs, distributed computing, metric compression, shortest path

\section{ACM Reference Format:}

Jason Li and Merav Parter. 2019. Planar Diameter via Metric Compression. In Proceedings of the 51st Annual ACM SIGACT Symposium on the Theory of Computing (STOC '19), June 23-26, 2019, Phoenix, AZ, USA. ACM, New York, NY, USA, 12 pages. https://doi.org/10.1145/3313276.3316358

\section{INTRODUCTION}

Computing the diameter of a graph is one of the most central problems in planar graph algorithms. In general weighted graphs, the best diameter algorithm is based on solving the All-Pairs Shortest Paths (APSP) problem. In planar graphs, however, the diameter can be solved considerably faster. In recent years there has been a substantial progress on this problem both for the exact as well as for the approximate setting.

Exact Diameter. Frederickson [10] gave the first $O\left(n^{2}\right)$ algorithm for the problem using APSP. A poly-logarithmic improvement was given by Wulff-Nilsen [39], providing the first indication that diameter is indeed easier than APSP. The question of whether one can compute the diameter in sub-quadratic time was one of the most important open problems in the area for quite some time. In a breakthrough result, Cabello [4], building upon the heavy machinery of Voronoi diagrams in planar graphs, presented the first truly sub-quadratic diameter algorithm that runs in time $\widetilde{O}\left(n^{11 / 6}\right) .{ }^{1}$ This works even for weighted and directed planar graphs. Soon after, by simplifying and extending the approach of Cabello, Gawrychowski et al. [14] improved the bound to $O\left(n^{5 / 3}\right)$, which is currently the state of the art. The techniques developed in $[4,14]$ led to subsequent improvements in the related setting of compact distance oracles $[7,8,15]$.

Approximate Diameter. In lack of truly efficient algorithms for diameter computation over the years, the area turned to consider the approximate setting. The most notable work in this context is by Weimann and Yuster [38] that provided the first $(1+\epsilon)$ approximation in time $\widetilde{O}\left(2^{O(1 / \epsilon)} \cdot n\right)$, hence linear for any constant $\epsilon$. Unlike the heavy machinery used by the exact algorithms, their approximate algorithm is based on a simple divide and conquer approach using shortest path separators. Ideas along this line were first introduced by [37] in the distance oracle setting. We elaborate more on this approach in the technical overview section. Chan and Skrepetos [6] combined the exact and approximate worlds by combining the algorithm of Weimann and Yuster [38] with the abstract Voronoi diagram tool of [4]. They achieve a randomized $(1+\epsilon)$ approximation in time $\widetilde{O}(\operatorname{poly}(1 / \epsilon) \cdot n)$. We note that one implication of our results is a considerably simpler deterministic

$\overline{{ }^{1} \text { As standard, } \widetilde{O}}$ is used to hide poly $\log n$ factors. 
"divide and conquer" algorithm for this problem that has the same time complexity of $\widetilde{O}(\operatorname{poly}(1 / \epsilon) \cdot n)$ but avoids the use of Voronoi diagrams.

\subsection{Distributed Algorithms for Planar Graphs}

Throughout, we use a standard message passing model of distributed computing called CONGEST[33]. The network is abstracted as an $n$-node graph $G=(V, E)$, with one processor on each network node. Initially, these processors do not know the graph. They solve the given graph problems via communicating with their neighbors. Communication happens in synchronous rounds. Per round, nodes can send $O(\log n)$-bit message to each of their neighbors.

The Distributed View Point. There is a subtle gap between the centralized and distributed point of views on planar graphs (and on global graph problems in general). In the centralized world, one usually thinks of the graph diameter $D$ in terms of the worst-case $\Omega(n)$ bound. For this reason, an $\sqrt{n}$-size separator is way more preferable over shortest path separators. In contrast, the prevalent viewpoint in distributed graph algorithms thinks of the graph's diameter as being a small number (independent of $n$ ). With this view, shortest-path separators are preferable over $\sqrt{n}$-size separators. This viewpoint has two justifications. First, as argued by Garay, Kutten, and Peleg in their seminal work [11, 30], real world networks usually do have small diameter. In addition, global graph problems admit a trivial $\Omega(D)$ lower bound in the distributed setting. Thus, a separator with $O(D)$ vertices is small w.r.t to the total round complexity.

Distributed Planar Graphs via Low-Congestion Shortcuts. The area of distributed planar algorithm was initiated by Ghaffari and Haeupler [18], who introduced the notion of low-congestion shortcuts. Roughly speaking, low-congestion shortcuts augment vertex disjoint subgraphs of potentially large diameter, with edges from the original graph in order to considerably reduce their diameter. Using this machinery, [18] has provided improved algorithms for MST and minimum-cut. Low-congestion shortcuts and their algorithmic applications have been studied extensively since then [22-24, 26, 31]. Recently, Ghaffari and Parter [20] presented a distributed construction of shortest path separator in nearly optimal time. We will use this algorithm extensively in our constructions.

Lack of Efficient Shortest Path Algorithms. Low-congestion shortcuts provide the fundamental communication backbone for many global graph problems. However, when it comes to distance related problems, the shortcuts by them-self seem to be insufficient. One of the key contributions in this paper is to provide a new recursive graph decomposition that preserves some distance related measures in each of the recursive pieces. This decomposition along with the low-congestion shortcuts provide the communication backbone for our algorithms. An exception for the above, is a recent work by Haeupler and $\mathrm{Li}$ [25] that used low-congestion shortcuts to compute $(\log n)^{O(1 / \epsilon)}$-approximate SSSP trees within $O\left(n^{\epsilon} \cdot D\right)$ rounds.

Distributed Shortest Paths in General Graphs. In contrast to planar graphs, the problem of distributed diameter computation in general graphs is fully understood. Frischknecht et al. showed a lower bound of $\widetilde{\Omega}(n)$ rounds that holds even for networks with constant diameter. Abboud, Censor-Hillel and Khoury [2] showed the same lower bound holds even if (i) the network is sparse (and with small diameter), or (ii) if we relax to an $(3 / 2-\epsilon)$ approximation in sparse graphs. A matching upper bound is known by Peleg, Roddity and Tal [34].

Unlike diameter, distributed shortest path computation for weighted graphs is a subject of an active research, attracting a lot of recent attention. Becker et al. presented a deterministic $(1+o(1))$ approximate shortest paths in $\widetilde{O}(D+\sqrt{n})$. Elkin [9] provided the first sublinear-time algorithm for exact single source shortest paths on undirected graphs. Huang et al. [27] presented an improved algorithm for the exact all pairs shortest paths. Recently, Ghaffari and Li [19] improved Elkin's result and presented an $\widetilde{O}\left(n^{3 / 4} \cdot D^{1 / 4}\right)$. This was improved even more recently by Forster and Nanongkai [29]. The lack of efficient distributed algorithms for these problems in general graphs provides the motivation for studying these problems in planar networks.

\subsection{Our Results}

We study the problem of distributed diameter computation (and related problems) by means of metric compression point of view. This approach is inspired by the approximate diameter algorithm of Weimann and Yuster [38], and the metric compression problem by Abboud at el. [3]. We start by defining the following problem, a special case of Abboud at el., which will underlie the combinatorial basis for our diameter computation.

\section{The Metric Compression Problem.}

Definition 1.1 (The OS Metric Compression Problem). In the OS (Okamura Seymour) Metric Compression Problem ${ }^{2}$ one is given an unweighted, undirected planar $n$-vertex graph $G$, a subset of sources $S \subseteq V$ of vertices lying on a single face in $G$, and a subset of target terminals $T \subseteq V$. The goal is to compute a bit string $\mathcal{S}$ that encodes all $S \times T$ distances. That is, there is a decoding function $f$ that given the encoding $\mathcal{S}$ and any two nodes $s, t \in S \times T$ returns the distance $d_{G}(s, t)$.

This problem can observed as a special case of the metric compression problem studied by Abboud et al. [3]. In particular, [3] considered an arbitrary subset $S \subseteq V$ with the objective to compress the $S \times S$ distances (rather than the $S \times T$ distances). Our formulation is motivated by diameter computation, where the set $S$ corresponds to the cycle separator of the graph and $T=V$, we then wish to compress the two sides across the cycle separator to speed up the computation of the diameter. We note the our solution is technically not related to [3]. In the latter, the main challenge is in handling the case where $S$ is not lying on a single face. In our case the challenge is in handling $S \times V$ distance rather than a small set of $S \times S$ distances. Indeed, our approach is different than that of [3], and it is based on VC-dimension type arguments. We are unaware of previous use of such arguments in the context of distance computation in planar graphs.

\footnotetext{
${ }^{2}$ The setting where the terminal vertices are on the boundary of a face is called an Okamura Seymour instance.
} 
Theorem 1.2 (Exact Compression). Given an $n$-vertex unweighted planar graph $G=(V, E)$, a set $S \subseteq V$ of sources lying consecutively on a single face, and a set $T \subseteq V$, there exists an algorithm that computes a compression of all $S \times T$ distances in $G$ using $\widetilde{O}\left(|S|^{3} \cdot D+|T|\right)$ bits.

For the case of weighted graphs, we also provide an $(1+\epsilon)$-approximate compression scheme:

Theorem 1.3 (Approximate Weighted Compression). Given an n-vertex weighted planar graph $G=(V, E, \omega)$ with aspect ratio $W$, a set $S \subseteq V$ of sources lying (not necessarily consecutively) on a single face, and set of terminal $T \subseteq V$, there exists an algorithm that computes a compression of $(1+$ $\epsilon)$-approximate $S \times T$ distances in $G$ using $\widetilde{O}((\operatorname{poly}(|S| / \epsilon)+$ $|T|) \log W)$ bits.

We complement these results by providing an efficient algorithm that computes the compressions in linear time (in the input and output size), this improves upon the naïve algorithm that takes $O(|S| \cdot n)$ time.

Distributed Diameter Computation. We are making a first step of progress on the distributed complexity of this classical problem, by presenting a poly $(D)$ round algorithm for $D$-diameter planar graphs. No sublinear round algorithm was known for the problem before.

Theorem 1.4 (Distributed Planar Diameter). Given an $n$-vertex unweighted, undirected planar graph with diameter $D$, there is a randomized distributed algorithm that computes the diameter in $\widetilde{O}\left(D^{5}\right)$ rounds, with high probability.

We also consider the problem of computing a $(1+\epsilon)$ approximation of the weighted diameter. Our end result is:

Theorem 1.5 (Approximate Weighted Compression). Given an n-vertex weighted, undirected planar graph with unweighted/hop diameter $D$ and aspect ratio $W$, for every $\epsilon \epsilon$ $(0,1]$, there exists a distributed approximate planar diameter algorithm that computes a $(1+\epsilon)$ approximation of the diameter in $\widetilde{O}\left(D^{2}+\operatorname{poly}(1 / \epsilon) \cdot D \cdot \log W\right)$ rounds, with high probability.

Distance Labels and (Exact) SSSP. It is well known that distributed shortest path computations in weighted graphs are considerably more challenging (and provably harder in general graphs). The above mentioned $(1+\epsilon)$ approximation results are based upon additional set of tools and constructions, most notably is a construction of an exact SSSP tree. This problem has attracted a lot of attention recently in general graphs.

Theorem 1.6 (ExAct SSSP Tree). There is a randomized distributed algorithm that given an n-node planar graph with unweighted diameter $D$, and vertex s computes an exact SSSP tree w.r.ts in $\widetilde{O}\left(D^{2}\right)$ rounds, with high probability.
Interestingly, this result does not use the low-congestion shortcut machinery. Instead, it is made possible due to our new recursive decomposition technique which preserves the unweighted diameter of each component throughout the recursion.

\section{TECHNICAL OVERVIEW}

Separators are subgraphs whose removal from the graph leaves connected components that are all a constant factor smaller than the initial graph. They provide the key tool in working with planar graphs (in the centralized setting). Typically, one desires the separator to be small, i.e, of size $\sqrt{n}$. In the distributed point of view, $D$ is typically considered to be smaller than $\sqrt{n}$ and thus in this context an $O(D)$-size separator is considered to be small. A celebrated result of Lipton and Tarjan [32] demonstrates the existence of a separator path in planar graphs. Their proof shows that ${ }^{3}$ :

For any SSSP tree $T$ in a planar graph $G$, there is a non-tree edge $e$ (possible $e \notin G$ ) such that the strict interior and strict exterior of the unique simple cycle $C$ in $T \cup\{e\}$ each contains at most $2 / 3 \cdot n$ vertices. Thus, $C$ forms a separator containing two shortest paths in $T$.

The High Level Approach for Diameter Computation. Our diameter computation is based on a common divide and conquer approach introduced by [37] using cycle separators.

In any independent step of the recursion, one is given a subgraph $G^{\prime} \subseteq G$ and the goal is to compute the largest distance in $G$ between vertex pairs in $G^{\prime}$. To do that, a cycle separator $C$ is computed in $G^{\prime}$

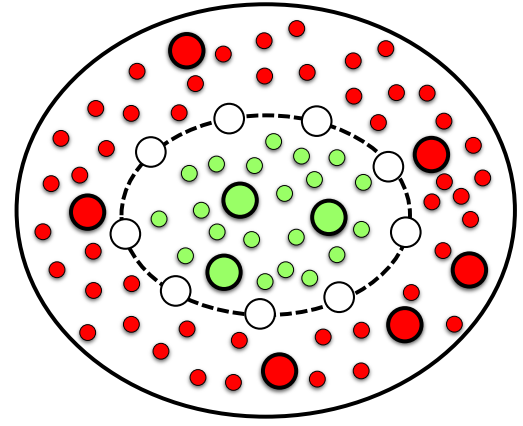
which subdivides $G^{\prime}$ into two subgraphs: the interior $G^{+}$and the exterior $G^{-}$. The key task is to compute the largest $G$-distance among all pairs that are separated by the separator $C$. Since $G^{+}$and $G^{-}$might have $\Omega(n)$ vertices, computing the distances between all pairs of vertices across the separator is inefficient both in the centralized and the distributed setting.

Our approach, inspired by [38], is based on compressing the two sides across the separator, $G^{+}$and $G^{-}$, into a small number of critical vertices $V^{*+} \subset G^{+}$and $V^{*-} \subset G^{-}$, such that the vertex pair of largest distance in $G^{+}, G^{-}$is contained $V^{*+}, V^{*-}$. We call these critical sets core-sets ${ }^{4}$. In the figure, shown is a cycle separator, and the two parts inside and outside the cycle, $G^{+}$and $G^{-}$. The core-sets are the filled large circles inside these regions. Having

\footnotetext{
${ }^{3}$ This was shown for a biconnected triangulated graph. However, since we can always augment a graph by adding additional edges to make it biconnected and triangulated (while keeping the vertex set to be the same), the statement holds for every graph. ${ }^{4} \mathrm{We}$ are aware to the fact that core-sets have similar yet a different context in the literature. We still use this term as it follows the same spirit of other existing core-sets.
} 
small size core-sets leads to a simple recursive scheme for diameter computation:

\section{Recursive Diameter via Metric Compression:}

- Compute a cycle separator $C$ in $G$, which decomposes $G$ into $G^{+}$and $G^{-}$(e.g., see [38] for the precise definition of $G^{+}$and $G^{-}$).

- Compute shortest path distances from $C$ to all vertices in G.

- Compute (via metric compression algorithm) the coresets $V^{*+} \subset G^{+}$and $V^{*-} \subset G^{-}$.

- Find the farthest pair in $V^{*+}$ and $V^{*-}$

- Recurse on $G^{+}$and $G^{-}$.

Figure 1: Simple Recursive Scheme by Compressing the Two Sides Across the Cycle Separator

\subsection{The Metric Compression Problem}

To provide the high level ideas, we start by considering an unweighted $D$-diameter planar graph $G$. Let $S \subseteq V(G)$ be a subset of vertices lying consecutively on a common face. For each vertex $v \in V$, define the distance tuple of $v$ to be its distances to $S$ stored as a tuple, defined as follows.

Definition 2.1 (Distance tuple). Let $G=(V, E)$ be a graph, and let $S=\left\{s_{1}, \ldots, s_{\ell}\right\} \subseteq V$ be a subset of vertices. The $S$-distance tuple of a vertex $v \in V$ denoted by tuple $\log _{S}(v)$ is the function that maps each vertex $v \in V$ to the vector of distances tuple $e_{S}(v)=$ $\left\langle d_{G}\left(s_{1}, v\right), \ldots, d_{G}\left(s_{\ell}, v\right)\right\rangle$. When the set $S$ is clear from the context, we simply use distance tuple and tuple(v).

To compress the distances, we will actually show that, perhaps surprisingly, there are only $O\left(|S|^{3} D\right)$ many possible distinct distance tuples. That is, when $n \gg O\left(|S|^{3} D\right)$, there are many repeated distance tuples among the vertices. This means that we can simply keep a table of the $O\left(|S|^{3} D\right)$ distance tuples, and store for each vertex $t \in T$ the index into the tuple, which has size $O(\log n)$. Therefore, the size of the compression is $O\left(|S|^{3} D+|T| \log (|S| D)\right)$. In Sec. 3 we show:

Theorem 2.2 (Theorem 1.2, Compression). Given an n-vertex unweighted planar graph $G=(V, E)$ and a set $S \subseteq V$ for sources lying consecutively on a single face, the number of distinct $S$-distance tuples (in $V$ ) is bounded by $O\left(|S|^{3} D\right)$.

We start by representing the distance tuple information as a set system. Let $S=\left\{s_{1}, \ldots, s_{\ell}\right\} \subseteq V$ be sorted according to their appearance on the face. For each $s_{i} \in S$, we define two sets $A_{i}^{\Delta}$ for $\Delta \in\{+,-\}$ containing vertices in $V$, where: $A_{i}^{-}=\left\{v \mid d_{G}\left(v, s_{i}\right)<\right.$ $\left.d_{G}\left(v, s_{i+1}\right)\right\}$ and $A_{i}^{+}=\left\{v \mid d_{G}\left(v, s_{i}\right)>d_{G}\left(v, s_{i+1}\right)\right\}$. For each vertex $v$, we define a set $F(v)=\left\{(i, \Delta) \mid v \in A_{i}^{\Delta}\right\}$. The set $F(v)$ can be considered as a weaker version of the distance tuple of $v$. Specifically, two vertices $v$ and $u$ with $F(v)=F(u)$, do not necessarily have the same distance tuples. We then define equivalence classes based on the $F(v)$ sets, where $u$ and $v$ are in the same equivalence class if $F(u)=F(v)$. Our goal is to show that there are poly $(|S|)$ equivalence classes. Assuming this, we are mostly done: once one knows $d_{G}\left(s_{1}, v\right)$ and $F(v)$, the entire distance tuple of $v$ is determined. As there are $D$ options for the starting value $d_{G}\left(s_{1}, v\right)$, the total number of tuples will be bounded by poly $(|S|) \cdot D$.

We will bound the number of equivalence classes using the VC dimension theory. In particular, we will use the well-known SauerShelah Lemma [35]:

Lemma 2.3 (Sauer-Shelah Lemma [35]). Let $\mathcal{F}=\left\{A_{1}, \ldots, A_{k}\right\}$ be a family of sets over universe of size $m$, and let $T$ be another set. We say that $\mathcal{F}$ shatters $T$ if for every subset $T^{\prime} \subseteq T$, there exists $A_{i} \in \mathcal{F}$ such that $A_{i} \cap T=T^{\prime}$. The $V C$-dimension of $\mathcal{F}$ is the largest set $T$ that can be shattered by $\mathcal{F}$. If the $V C$-dimension of $\mathcal{F}$ is $k$, then $|\mathcal{F}|=O\left(m^{k}\right)$.

In our setting, $\mathcal{F}$ contains one representative set $F(v)$ from each of the equivalence classes. Thus the size of $\mathcal{F}$ is the same as the number of equivalence classes. The universe is $\mathcal{S}=\left\{\left(s_{i}, \Delta\right) \mid s_{i} \in\right.$ $S, \Delta \in\{+,-\}\}$. Thus the universe size is $2|S|$. Suppose towards contradiction that the VC-dimension of $\mathcal{F}$ is four. By the Sauer-Shelah Lemma, we get that there is a set $T=\left\{\left(i_{1}, \Delta_{i_{1}}\right),\left(i_{2}, \Delta_{i_{2}}\right),\left(i_{3}, \Delta_{i_{3}}\right)\right.$, $\left.\left(i_{4}, \Delta_{i_{4}}\right)\right\}$ that is shattered by $\mathcal{F}$. We first argue that we can assume w.l.o.g. that $i_{1}<i_{2}<i_{3}<i_{4}$. We then consider two subsets $T_{1}=\left\{\left(i_{1}, \Delta_{i_{1}}\right),\left(i_{3}, \Delta_{i_{3}}\right)\right\}$ and $T_{2}=\left\{\left(i_{2}, \Delta_{i_{2}}\right),\left(i_{4}, \Delta_{i_{4}}\right)\right\}$, and show that it cannot be that there are two vertices $t_{1}, t_{2}$ such that $F\left(t_{1}\right) \cap T=T_{1}$ and $F\left(t_{2}\right) \cap T=T_{2}$. This implies that the VC dimension of $\mathcal{F}$ is at most 3 , and thus that $|\mathcal{F}|=O\left(|S|^{3}\right)$. The proofs of these arguments are quite tedious, as we need to consider many cases, but aside from that, each of the cases is rather easy and follows immediately from the planar embedding.

Fast Computation. We continue with the unweighted case of $D$ diameter planar graph $G=(V, E)$ with $S \subseteq V$ sources on a face. A naïve computation applies a SSSP (single-source shortest-path) algorithm for each $s \in S$, which takes $O(|S| \cdot n)$ time. Our goal is compute all distance tuples in time $\widetilde{O}\left(n+|S|^{4} \cdot D\right)$ time.

At a high level, we will follow the multiple-source shortest path (MSSP) algorithm with all sources lying on a common face, from [28], while maintaining hashes of distance tuples. Observe that we cannot explicitly maintain the size- $|S|$ distance tuple for each vertex, since that is $n \cdot|S|$ integers, which exceeds the promised time bound of $\widetilde{O}\left(n+|S|^{4} \cdot D\right)$ if $|S|$ is large (say, $\left.n^{\Omega(1)}\right)$. Therefore, we maintain hashes of size $O(\log n)$ instead. The tricky part is to efficiently update the distance tuples while running the MSSP algorithm. Our hash function is motivated by Rabin-Karp string hashing. For a distance tuple $\mathbf{d}=\left(d_{1}, \ldots, d_{\ell}\right)$, we define its hash value under base $b$ and modulus $p$ (for $p$ prime) as $h(\mathbf{d}, b, p):=\sum_{i=1}^{\ell} d_{i} b^{i} \bmod p$. Clearly, if two distance tuples are equal, then their hash values under the same base and modulus are equal. We then claim that for two distinct distance tuples, their hash values are likely to be different under a random base, as long as the modulus is large enough.

The MSSP algorithm begins with computing the SSSP tree on the first source $s_{1}$. It then travels along the face segment in the $\operatorname{order}\left(s_{2}, s_{3}, \ldots, s_{\ell}\right)$, temporarily setting each $s_{j}$ as the source, while maintaining a dynamic forest $F$ of values, one for each vertex in $V$. (It also maintains a dynamic forest on the dual graph, but we do not need to discuss that here.) Our algorithm will maintain another dynamic forest $F^{\prime}$ on the vertices in $V$ that is updated alongside 
the MSSP algorithm, so that at the end, the value at each vertex $v$ is precisely the hash value of its tuple. The detailed description is in the full version.

The weighted case will be similar to the unweighted one with a key crucial difference. Here, since we are looking for approximate distances, what we need is not a hash function, but a "clustering" function that groups together vertices whose distance tuples are close together (say, in $\ell_{2}$-distance). For this, we will use the Johnson-Lindenstrauss (JL) dimension reduction scheme. The complete algorithm appears in the full version.

Implications to Distance Oracles. Bounding the number of tuples by $O\left(|S|^{3} D\right)$, immediately leads to an efficient compact distance oracles scheme for maintaining $S \times T$ distances. The oracle will contain the $O\left(|S|^{3} \cdot D\right)$ distinct distance tuples using $O\left(|S|^{4} \cdot D \log n\right)$ bits. Next, the distance tuple of each vertex $t \in T$ can be encoded with $O(\log (|S| D))$ bits (i.e., encoding the index of the tuple of $t$ in the list of all tuples). Given a query $s, t \in S \times T$, the oracle can compute $d_{G}(s, t)$ by extracting this information for the tuple of $t$ in constant time. The preprocessing time of the construction is linear (in the size of the oracle), due to the fast computation of the tuples.

Implications to Diameter Computation. In the context of diameter computation, $S$ will be the cycle separator of size $O(D)$. To compute the core-set $V^{*+}$, we simply take one representative vertex in $G^{+}$for each of the poly $(D)$ equivalence classes. The core-set $V^{*-}$ is defined analogously. This leads immediately to an $\widetilde{O}(\operatorname{poly}(D) \cdot n)$ time deterministic algorithm for the unweighted case, by plugging it in the recursive procedure described before. Similar bounds are obtained by [6], using the heavy machinery of abstract Voronoi diagram.

The Weighted Case. For the weighted case, in the full version, we consider an $(1+\epsilon)$-approximate compression scheme that maintains a $(1+\epsilon)$ approximation for all $S \times T$ distances. Here, we first provide a compression scheme with an additive error with respect to the weighted diameter of the graph. We then reduce the multiplicative error case to the additive via the use of low-diameter decompositions. We remark that for the purpose of diameter computation, the additive compression scheme suffices. The additive approximate compression is based on the notion of (additive) close and (additive) core-set.

Definition 2.4 ( $\delta$-Additive Close). Let $G=(V, E)$ be a graph, let $S=\left\{s_{1}, \ldots, s_{\ell}\right\} \subseteq V$ be a subset of vertices. Two vertices $u, v \in V$ are $\delta$-additive close with respect to $S$ if

$$
\left|d\left(u, s_{i}\right)-d\left(v, s_{i}\right)\right| \leq \delta \quad \forall i \in[\ell] .
$$

Definition 2.5 (Additive Core-Set). Let $G=(V, E)$ be a graph, let $S=\left\{s_{1}, \ldots, s_{\ell}\right\} \subseteq V$ be a subset of vertices, and let $\delta \geq 0$ be an additive error parameter. A subset $V^{\prime} \subseteq V$ is a $\delta$-additive core-set with respect to $S$ if for all vertices $v \in V$, there exists a vertex $v^{\prime} \in V^{\prime}$ that is $\delta$-additive close to $v$ w.r.t $S$.

Our goal will be to prove the existence of a core-set of size $\operatorname{poly}(|S| / \epsilon)$.

Theorem 2.6. Let $G=(V, E)$ be a weighted graph, and let $d>0$ be a parameter. Let $S=\left(s_{1}, \ldots, s_{\ell}\right)$ be a sequence of points on a common face arranged in cyclic order, such that the distance between any two consecutive points is at most $d$. Then, there exists a $\delta$-additive core-set of size $O\left(\ell^{6}(d / \delta)^{4}\right)$.

We will define sets $A_{i}^{\Delta}$ similarly to the ones in the unweighted case, but with more values of $\Delta$. In particular, we will consider all multiples of $\delta / \ell$ from roughly $-d$ to $d$. We will then apply a similar $\mathrm{VC}$ dimension argument as in the unweighted case, but with several subtleties as the weighted sets $A_{i}^{\Delta}$ are more involved.

\subsection{Distributed Tools and Unweighted Diameter}

The challenge. The efficiency our of recursive diameter computation critically depends on the size of the separator. Recall that also the bound on the size of the core-sets is a function of the separator size and the diameter of the graph. [20] provided an $\widetilde{O}(D)$-round algorithm that computes a shortest path separator, thus a separator of size $O(D)$. In our algorithm the separator should be computed recursively, until all components are sufficiently small. The key challenge is that already after the first computation, once we remove the vertices of the separator from the graph, the diameter of each of the components might be $\Omega(n)$. We note that although the DFS construction of [20] also applied the separator algorithm in a recursive manner, for their purposes it was sufficient for the separator to be a path, and its length could be arbitrarily large. In our setting in contrast, we need to come up with a different recursive scheme that preserves the diameter of the subgraphs throughout all recursion layers. This is our motivation for defining the bounded diameter decomposition.

Bounded Diameter Decomposition (BDD). Informally, the bounded diameter decomposition is described by a recursive procedure that given a subgraph $G^{\prime}$ of diameter $D^{\prime}$, breaks down $G^{\prime}$ into small components $G_{1}^{\prime}, \ldots, G_{k}^{\prime}$ each with at most $\left|G_{j}^{\prime}\right| \leq\left|G^{\prime}\right| / c$ vertices, for some constant $c$, such that:

- The diameter of each $G^{\prime}$ is bounded as a function of $D^{\prime}$.

- Each edge $e \in G^{\prime}$ appears on a small number of $G_{j}^{\prime}$ subgraphs.

The first property is important for being able to compute an $O(D)$ separator recursively. The second property is important for parallelizing the computation on all the components (i.e., via the randomdelay approach). Our formal definition of BDD is in fact considerably more delicate for the following reasons. Let $T$ be a BFS tree on which the shortest-path separator is computed in the first recursion level. Let $S$ be the balanced cycle separator ${ }^{5}$ of $G$. To define the child components of $G$, there are two options. The first defines the child components in $G \backslash S$. while this satisfies the second property, it might violate the first property. Alternatively, one might first define the interior and exterior subgraphs w.r.t the cycle $S$ and then augment both parts with the vertices in $S$. This satisfies the first property, but now as $S$ is added to both parts, an edge might appear later on, on many subgraphs in the same recursion layer.

To get out of this impasse, our technique adds segments of $S$ to each of the components, while guaranteeing that the second property holds. Since we do not add $S$ entirely to both parts, this might increase the diameter of the components. We then show that this increase is rather controlled, by an additive $+D$ term, in each

${ }^{5}$ This cycle separator might contain a non- $G$ edge, which we simulate as a virtual edge. 
recursive level. Thus, after all $O(\log n)$ recursion levels, the diameter is still bounded by $O(D \log n)$. This recursive decomposition continues until the components have size $O(D \log n)$. A useful property of the components is they have an $O(D \log n)$-depth spanning tree that contains at most $O(\log n)$ edges that are not in the BFS tree. This property will become useful in the weighted setting.

Handling 1-Connected Subgraphs. The basic separator algorithm of [20] (which we will use throughout) requires that the boundary of each face is a simple cycle which indeed holds for biconnected subgraphs. Since we need to compute the separator recursively, even if the original input graph is biconnected, after one recursive layer it can become 1-connected. [20] handled this by computing the biconnected components of the graph and solving the problem for each piece separately. We take a rather different approach that allows us to simulate the separator algorithm for biconnected graphs in 1-connected graphs with a small overhead in the number of rounds. This reduction is based on adding "virtual" edges to the 1connected graph in order to make it biconnected. We then simulate these virtual edges by providing short and low-congestion paths between the endpoints of the virtual edges. This tool of distributed biconnected augmentation might provide a cleaner and more general way to handle 1-connected graphs.

Exact Distance Labels. To facilitate the recursive diameter computation, we first compute exact labels of $\widetilde{O}(D)$-bits. Our labels are based on the well-known scheme of Gavoille et al. [13] that has the following recursive structure. Each label $L_{G}(v)$ consists of (i) the $G$-distances from $v$ to all the vertices in the separator $S$ of $G$, (ii) the component ID of $v$ in $G \backslash S$, and (iii) the label $L_{G^{\prime}}(v)$, where $G^{\prime}$ is the component of $v$ in $G \backslash S$.

Our goal is to compute these labels in a top-down manner over the recursion tree of the BDD decomposition. The key challenge here is that unlike the recursion of [13] in which the child components are vertex-disjoint, here subgraphs are not vertex disjoint, and a vertex might belong to many subgraphs in the same recursion level. The fact that the components of [13] are disjoint, implies that a vertex belongs to $O(\log n)$ components in total, thus keeping the label small. In our case, we will not be able to keep a sub-label of $v$ for each of the subgraphs for which it belongs. To handle that, we will use the fact that the components in the recursive partitioning of [13] are fully contained in the components of the BDD decomposition.

Distributed Unweighted Diameter. For the sake of explanation, we sketch here a poly $(D)$-round algorithm. Obtaining the $\widetilde{O}\left(D^{5}\right)$-round algorithm calls for various combinations of techniques that shave off some of the $D$-factors. We first compute the distance labels of the vertices. The subsequent diameter computation works in a bottomup manner on the BDD decomposition tree. The invariant that we will maintain is that in step $i$, all vertices in the $(D-i+1)$-level subgraphs $G^{\prime}$ (in the BDD decomposition) have already computed the largest distance in $G$ (and not in $G^{\prime}$ ) over all vertex pairs in $G^{\prime}$ The leaf components of the BDD decomposition have $O(D)$ many vertices, and thus every vertex can collect the distance label of all the vertices in its leaf components in $\widetilde{O}\left(D^{2}\right)$ rounds. Since all these subgraphs are almost-edge disjoint, this computation can be done in parallel in all of these subgraphs.
Consider the $i^{t h}$ phase of this process. For every $(D-i+1)$-level subgraph $G^{\prime}$, there are two options. Either the farthest pair $u, v$ in $G^{\prime}$ is contained in one of the child components, or that $u$ and $v$ are in different child components. The interesting case is the second one. Since $u$ and $v$ are in different component in the BDD, they are separated by the shortest path separator $S$ of $G^{\prime}$. To compute the largest distance between vertices in different child components, we first let all vertices in the separator send their label to a global leader in $G^{\prime}$. This is a total of $\widetilde{O}(|S| \cdot D)$ bits of information, and since $D\left(G^{\prime}\right)=O(D \log n)$, using standard pipeline procedure it can be implemented in $O\left(D^{3}\right)$ rounds. At this points, all the vertices in $G^{\prime}$ can compute their distance tuple with respect to $S$. Thanks to our metric compression solution, there are $O\left(D^{3}\right)$ distinct distance tuples. The last step is to aggregate all these tuples at a leader in $G^{\prime}$. This can be done in $O\left(D^{6}\right)$ rounds, but in our algorithm we do it more efficiently in $\widetilde{O}\left(D^{5}\right)$ rounds by compressing each distance tuple into $O(\log n)$ bits. Once the leader in $G^{\prime}$ receives all the distance tuples, it has all the information to compute the distance in $G$ between each pair in different child components. This holds since every such $u$-v shortest-path must intersect $S$ in some vertex $s$, thus $d_{G}(u, v)=d_{G}(u, s)+d_{G}(s, v)$, these distances are contained in the distance tuple information.

\subsection{Distributed Weighted Diameter}

The computation of $(1+\epsilon)$ approximate diameter is considerably more involved. It consists of several steps, and thus intermediate results (e.g., SSSP tree) which are important on their own.

Step (1): SSSP via Distance Labels. The major step here is the computation of exact distance labels with $\widetilde{O}(D)$-bits. Once we compute such labels, an SSSP can be easily defined: the source node $s$ sends its label $L_{G}(s)$ on the BFS tree to all the vertices. This allows each vertex $v$ to compute $d_{G}(s, v)$ based on its own label $L_{G}(v)$, and label of $L_{G}(s)$ of the source. By exchanging these distances with their neighbors, every vertex can compute its parent in the SSSP tree. Since the label size is $\widetilde{O}(D)$, we get that given exact labels, the SSSP can be computed within extra $\widetilde{O}\left(D^{2}\right)$ rounds.

From that point on, we focus on labels computation. Interestingly, we will compute these labels using the unweighted communication backbone of the unweighted BDD, namely, a BDD on a BFS tree. The key difference from the unweighted distance labels is that here we cannot afford to compute an SSSP from each of the separator vertices. Recall that efficient SSSP is the reason for computing this labels from first place. The key idea of our algorithm is it "morally" applies the scheme of Gavoille et al. [12] but in a bottom-up rather than a top-down manner.

We will start from the leaf components which have $O(D)$ vertices. In each such leaf component $G^{\prime}$, a vertex $v$ can collect the entire subgraph and locally compute a label $L_{G^{\prime}}(v)$ that consists of all its distances to the vertices in $G^{\prime}$. We now work from the leaf up, and consider the level- $i$ components in the BDD recursion tree. Consider a subgraph $G^{\prime}$ and its children components $G_{1}^{\prime}, \ldots, G_{k}^{\prime}$ in level $i+1$. Let $S$ be the separator of $G^{\prime}$ (based on which the child components are defined). By the induction assumption, we assume that each vertex $v$ in $G_{j}^{\prime}$ has already computed its label $L_{G_{j}^{\prime}}(v)$. Thus by the recursive label's structure of Gavoille et al. to compute $L_{G^{\prime}}(v)$, it is sufficient to augment the sub-label $L_{G^{\prime}}(v)$ with the distances (in 
$\left.G^{\prime}\right)$ to each of the separator vertices in $S$. By letting each separator vertex $s \in S$ send their labels $\left\{L_{G_{j}^{\prime}}(s), \mid s \in G_{j}^{\prime}\right\}$ to all vertices in $G^{\prime}$, every vertex $v \in G_{j}^{\prime}$ for every $j$ has now the sufficient information to compute its distance to $S$ in $G^{\prime}$ (using also its own label $L_{G_{j}^{\prime}}(v)$ ). In the analysis, we show that each separator vertex appears on at most two child components of $G^{\prime}$ (but potentially on many other subgraphs in this level), thus the total amount of information to be sent is $\widetilde{O}\left(D^{2}\right)$. Since a separator vertex in level $i$ might appear in many subgraphs of levels $j \geq i$, we will mimic again the Gavoille et al. label structure, and shorten the labels of vertices once we get to a level in which they are part of the separator, this step is vital to keep the labels short.

Step (2): BDD Decomposition on the SSSP Tree. At this point, we already have all the ingredients necessary for an $(1+\epsilon)$ approximation in $\operatorname{poly}(D) \cdot \operatorname{poly}(1 / \epsilon)$ rounds. Such an algorithm can be obtained by applying the exact same algorithm for the unweighted diameter, with the only difference is that the core-set size will be $\operatorname{poly}(D) \cdot \operatorname{poly}(1 / \epsilon)$.

Obtaining a better bound of $\widetilde{O}\left(D^{3}\right)+D^{2} \cdot \operatorname{poly}(\log n / \epsilon)$ calls for several improvements. First, we apply the BDD decomposition on an SSSP tree rather than on the BFS tree. Then, instead of computing the tuples w.r.t all the vertices on the cycle separator, we will select $O(\log n / \epsilon)$ portal nodes on this cycle (as in [38]). For this approach to work, the portals cannot be selected arbitrarily, but rather should be selected carefully on a shortest-path separator. Since the separator computed on a BFS tree is no longer a shortest-path in a weighted graph, we need to apply the BDD scheme on the SSSP tree. This will guarantee that the separator computed in each recursive layer will consist of a concatenation of $O(\log n)$ shortest path segments. We will then be able to mark $O(1 / \epsilon)$ portal vertices on each such segment, and ignore the remaining vertices on the separator.

Computing a BDD on the SSSP tree brings along several complications. The major one is that the unweighted diameter of each component might be very large. The standard remedy for these kind of problems is low-congestion shortcuts. However, as the components in each recursion level are not vertex disjoint, an additional argument is required in order to be able to compute the low-congestion shortcuts. For that purpose, we define the BDD in a more careful manner that guarantees the following: each edge $e \in G$ might appear on at most two components in the same level one from each side of that edge. This allows us to apply the graph simulation technique of [20]. This technique projects $G$ into a different graph $G^{\prime}$ that contains the vertices of $G$ plus additional vertices. The subgraphs in the BDD level are mapped in $G^{\prime}$ to vertex disjoint subgraphs, which allows safe application of low-congestion shortcuts in $G^{\prime}$. The vertices will then simulate the low-congestion computation in $G^{\prime}$ and will translate it back to the edge of $G$.

Step (3): Recursive Diameter Computation. The algorithm has the same high level structure as for the unweighted, only that is works on the weighted BDD (i.e., BDD on the shortest-path tree) and uses approximate core-set with respect to a collection of $O(\log n / \epsilon)$ portals on the separator (defined as in [38]). In each independent level of the recursion, given a component $G^{\prime}$, compute a cycle separator $S$ that defines the subgraphs $G^{+}$and $G^{-}$. We then compute exact distance labels in $G^{+}$and in $G^{-}$using a total of $\widetilde{O}\left(D^{3}\right)$ rounds for all subgraphs in that level. Next, we restrict attention only to $O(\log n / \epsilon)$ portals on the separator of $G^{\prime}$, and compute the $(1+\epsilon)$ approximate core-sets in $G^{+}$and $G^{-}$. The portals then send their exact labels (in $G$ ) over the low-congestion shortcuts of $G^{\prime}$. Since each edge of these shortcuts appears on $\widetilde{O}(D)$ subgraphs, the total amount of information that we pass through an edge is poly $(\log n / \epsilon) \cdot D^{2}$. The method of random delay then allows us to work on all components in parallel with a total round complexity of $\widetilde{O}\left(D^{3}\right)+\operatorname{poly}(\log n / \epsilon) \cdot D^{2}$.

\subsection{Preliminaries}

Graph Notations. For a weighted graph $G=(V, E, \omega)$, let $d_{G}(u, v)$ be the total weight of the shortest path between $u$ and $v$ in $G$. When $G$ is clear from the context, we may omit it. For a tree $T \subseteq G$, let $T(z)$ be the subtree of $T$ rooted at $z$, and let $\pi(u, v, T)$ be the tree path between $u$ and $v$, when $T$ is clear from the context, we may omit it and simply write $\pi(u, v)$. For a subset of vertices $S_{i} \subseteq V(G)$, let $G[S]$ be the induced subgraph on $S$.

Planar Embeddings. The geometric planar embedding of graph $G$ is a drawing of $G$ on a plane so that no two edges intersect. A combinatorial planar embedding of $G$ determines the clockwise ordering of the edges of each node $v \in G$ around that node such that all these orderings are consistent with a plane drawing (i.e., geometric planar embedding) of G. Ghaffari and Haeupler [17] gave a distributed algorithm that computes a combinatorial planar embedding in $O(D \min \{\log n, D\})$ rounds, where each node learns the clockwise order of its edges.

Low-Congestion Shortcuts. In a subsequent paper [18], Ghaffari and Haeupler introduced the notion of low-congestion shortcuts, which provides as basic communication backbone in many planar algorithms. The definition is as follows.

Definition 2.7. ( $\alpha$-congestion $\beta$-dilation shortcut) Given a graph $G=(V, E)$ and a partition of $V$ into disjoint subsets $S_{1}, \ldots, S_{N} \subseteq V$, each inducing a connected subgraph $G\left[S_{i}\right]$, we call a set of subgraphs $H_{1}, \ldots, H_{N} \subseteq G$, where $H_{i}$ is a supergraph of $G\left[S_{i}\right]$, an $\alpha$-congestion $\beta$-dilation shortcut if we have the following two properties: (1) For each $i$, the diameter of the subgraph $H_{i}$ is at most $\beta$, and (2) for each edge $e \in E$, the number of subgraphs $H_{i}$ containing $e$ is at most $\alpha$.

Ghaffari and Haeupler [18] proved the existence of almost optimal low-congestion covers, as well as providing efficient algorithm to compute them.

FACt 2.8 (Low-Congestion Shortcuts [20]). Any partition of a D-diameter planar graph into disjoint subsets $S_{1}, \ldots, S_{N} \subseteq V$, each inducing a connected subgraph $G\left[S_{i}\right]$, admits an $\alpha$-congestion $\beta$-dilation shortcut where $\alpha=O(D \log D)$ and $\beta=O(D \log D)$. Moreover, there is a randomized algorithm that computes these shortcuts within $\tilde{O}(D)$, with high probability.

Distributed Scheduling of Algorithms. Our algorithms are based on recursive graph decomposition, where in every level of the recursion we will need to work in parallel on several subgraphs. Since the CONGESTmodel allows sending only $O(\log n)$ bits on each edge per round, we will use the scheduling framework of [16]. In our context, this framework implies that if each edge appears on a small number of subgraphs in each recursion level, then all the algorithms 
(one per subgraph) can be scheduled within almost same number of rounds as a single algorithm.

FACt 2.9 (Scheduling, [16]). Given a sequence of algorithms $\mathcal{A}_{1}, \ldots, \mathcal{A}_{k}$, each taking at most $\mathrm{d}$ rounds, and where for each edge, at most $\mathrm{c}$ messages are sent through it in total over all these algorithms, then all algorithms can run in total of $\mathrm{O}(\mathrm{d}+\mathrm{c})$ rounds.

The Shortest Path Separator Algorithm by [20]. Throughout we will also make an extensive use of the separator algorithm by Ghaffari and Parter [20].

Fact 2.10 (Distributed Shortest-Path Separator, [20]). There is a randomized algorithm that given a D-diameter graph computes a shortest-path separator in $\widetilde{O}(D)$ rounds, with high probability.

The algorithm of [20] gets as input a biconnected graph $G$, and a spanning tree $T \subset G$. It outputs a cycle consisting of a tree path in $T$ plus one additional edge (possibly not in $G$ ). In the high-level the algorithm computes this separator by considering the dual-tree $T^{\prime}$ of $T$. The nodes of this dual tree are the faces of $G$, and two dualnodes are connected in $T^{\prime}$ if their faces share an non-tree edge $e \notin T$. The dual tree is rooted at the outface, and each dual-node $v^{\prime}$ is given a weight as follows: consider the superface obtained by merging all faces (dual-nodes) in the subtree of $T^{\prime}$ rooted at $v$; then the weight of $v^{\prime}$ is the number of nodes on its superface boundary plus the number of nodes inside the superface. The algorithm first compute a $(1+\epsilon)$ approximation of all dual nodes in $T^{\prime}$. This computation is done on the dual tree whose vertices and edges are not part of $G$ and thus call for special tool. Once all weights are computed, the algorithm first attempts at finding a balanced dual-node, a dualnode whose weight is in $[n /(3(1+\epsilon)), 2(1+\epsilon) n / 3]$. If such balanced dual-node than the boundary of its superface is the fundamental cycle separator (all the edges of this cycle are in $G$ ). Otherwise, there must be a critical dual-node such that its own weight is large but the weight of each of its children in $T^{\prime}$ is small. In this case, the algorithm mimics Lipton and Tarjan algorithm by roughly speaking "triangulating" the face of this critical dual node. In this latter case, the cycle separator contains one edge that is not in $G$.

Road-Map. We start by presenting the metric compression problem in Sec. 3. This provides the combinatorial basis for our diameter algorithms. Sec. 4 presents the key tool of bounded diameter decomposition and computation of exact diameter in unweighted graphs. In the full version, we describe the construction of exact distance labels in weighted graphs and a construction of SSSP (singe source shortest path) tree. Finally, we conclude the computation of $(1+\epsilon)$ approximation for the diameter in weighted graphs.

\section{THE METRIC COMPRESSION PROBLEM}

We consider the setting of exact compression for unweighted graphs. In the full version, we extend the result to the $(1+\epsilon)$-approximate compression for weighted graphs.

Exact Compression for Unweighted Graphs. Let $G$ be a planar graph under some planar embedding, and let $S$ be a subset of vertices lying consecutively on a common face. For each vertex $v \in V$, define the distance tuple of $v$ to be its distances to $S$ stored in a tuple, defined formally as follows.
Definition 3.1 (Distance tuple). Let $G=(V, E)$ be a graph, and let $S=\left\{s_{1}, \ldots, s_{\ell}\right\} \subseteq V$ be a subset of vertices. The $S$-distance tuple of a vertex $v \in V$ denoted by $\operatorname{tuple}_{S}(v)$ is the function that maps each vertex $v \in V$ to the vector of distances tuple ${ }_{S}(v)=$ $\left\langle d_{G}\left(s_{1}, v\right), \ldots, d_{G}\left(v, s_{\ell}\right)\right\rangle$. When the set $S$ is clear from the context, we simply use distance tuple and tuple(v).

To compress the distances, we will actually show that, perhaps surprisingly, there are only $O\left(|S|^{3} D\right)$ many possible distinct distance tuples. That is, if $n>>|S|^{3} D$, then there are many repeated distance tuples among the vertices. This means that we can simply keep a table of the $O\left(|S|^{3} D\right)$ distance tuples, and store for each vertex $v \in T$ the index into the tuple, which has size $O(\log n)$. Therefore, the size of the compression is $O\left(|S|^{3} D+|T| \log n\right)$. We will show:

Theorem 3.2 (Theorem 1.2, Compression). Given an $n$-vertex unweighted planar graph $G=(V, E)$ and a set $S \subseteq V$ for sources lying consecutively on a single face, the number of distinct $S$-distance tuples is bounded by $O\left(|S|^{3} D\right)$.

Our proof consists of two main steps. First, we define an alternative representation of distance tuples that utilizes the definition below.

Definition 3.3. For each $i \in[\ell-1]$ and $\Delta \in\{-1,0\}$, define the set $A_{i}^{\Delta}:=\left\{v \in V: d\left(v, s_{i}\right) \leq d\left(v, s_{i+1}\right)+\Delta\right\}$. Define the family

$$
\mathcal{F}(S):=\left\{\left\{(i, \Delta): v \in A_{i}^{\Delta}\right\}: v \in V\right\}
$$

We show that, modulo a factor of $O(D)$, our task of bounding the number of distance tuples reduces to bounding the size of $\mathcal{F}(S)$. Second, we prove the size bound $|\mathcal{F}(S)| \leq O\left(|S|^{3}\right)$ using a VCdimension argument. In particular, we show that the set system represented by $\mathcal{F}(S)$ has VC dimension at most 3. Combining these two steps proves the desired $O\left(|S|^{3} D\right)$ bound on distinct distance tuples.

\subsection{Reduction to VC Dimension Argument}

We now proceed with the technical details, beginning with the alternative representation step. Fix an arbitrary $r \in[\ell]$, and define $[D]_{0}:=\{0,1,2, \ldots, D\}=\{0\} \cup[D]$. Our domain will be $[D]_{0} \times$ $\mathcal{F}(S)$, and each vertex $v \in V$ will be represented by the tuple $\left(d\left(v, s_{r}\right),\left\{(i, \Delta): v \in A_{i}^{\Delta}\right\}\right) \in[D]_{0} \times \mathcal{F}(S)$. Here, we use the fact that the graph diameter is at most $D$, so $d\left(v, s_{r}\right) \in[D]_{0}$. We first prove the following claim, which establishes a surjective map from $[D]_{0} \times \mathcal{F}(S)$ to the set of distinct distance tuples. This bounds the number of distinct distance tuples by the size of the domain $[D]_{0} \times \mathcal{F}(S)$.

CLAIM 3.4. Let $r$ be any integer in $[\ell]$. For each vertex $v \in V$, its distance tuple is determined by the value of $d\left(v, s_{r}\right)$ and which sets $A_{i}^{\Delta}$ contain $v$. More formally, there is a function $f$ from $\mathbb{N} \times 2^{[\ell] \times\{-1,0\}}$ to the set of $S$-distance tuples such that for all $v$, the distance label of $v$ is precisely $f\left(d\left(v, s_{r}\right),\left\{(i, \Delta): v \in A_{i}^{\Delta}\right\}\right)$.

Proof. Fix a vertex $v \in V$; we will reconstruct the distance tuple for $v$ based on $d\left(v, s_{r}\right)$ and which sets $A_{i}^{\Delta}$ contain $v$. The value $d\left(v, s_{r}\right)$ is already known; we now proceed to calculate $d\left(v, s_{i}\right)$ for $i<r$. First, note that for all $i \in[\ell-1]$, by the triangle inequality and the fact that $s_{i}, s_{i+1}$ are distance 1 apart, we have 
$d\left(v, s_{i}\right)-d\left(v, s_{i+1}\right) \in\{-1,0,1\}$. Whether or not $v \in A_{r-1}^{-1}$ determines whether or not $d\left(v, s_{r-1}\right)-d\left(v, s_{r}\right)=-1$. If so, then we must have $d\left(v, s_{r-1}\right)=d\left(v, s_{r}\right)-1$, and we are done. Otherwise, $d\left(v, s_{r-1}\right)-d\left(v, s_{r}\right)$ is either 0 or 1 . It must be 0 if $v \in A_{i}^{0}$, and otherwise, it must be 1 , so in either case, we are done.

We can now proceed inductively from $r-1$ to 1 : knowing $d\left(v, s_{i}\right)$ for $i \in[2, r]$, we can deduce $d\left(v, s_{i-1}\right)$. This gives us all distances $d\left(v, s_{i}\right)$ for $i \in[r]$. For the remaining distances $d\left(v, s_{i}\right)$ for $i \in$ $[r+1, \ell]$, we can proceed analogously.

3.1.1 VC Dimension Argument. Since $[D]_{0}$ has size $D+1$, to bound the domain size by $O\left(|S|^{3} D\right)$, it suffices to bound $\mathcal{F}(S)$ by $O\left(|S|^{3}\right)$. We will prove that the VC-dimension of $\mathcal{F}(S)$ is at most 3, and then apply the well-known Sauer's Lemma.

Definition 3.5 (VC Dimension of a Set System). Let $X$ be a set of elements, called the universe. A family $\mathcal{F}$ of subsets of $X$ has $V C$ dimension $d$ if $d$ is the largest possible size of a subset $Y \subseteq X$ satisfying the following property: for any subset $Y^{\prime} \subseteq Y$, there exists subset $F \in \mathcal{F}$ such that $Y \cap F=Y^{\prime}$.

TheOREM 3.6 (SAUER'S LEMMA). Let $X$ be a set of elements. If a family $\mathcal{F}$ of subsets of $X$ of VC dimension d, then $|\mathcal{F}|=O\left(|X|^{d}\right)$.

We now proceed with the VC dimension argument. For convenience, we redefine $\mathcal{F}(S)$ in the statement of the theorem.

Theorem 3.7 (Bounded VC-Dimension). Let $G=(V, E)$ be an unweighted planar graph, and let $S:=\left(s_{1}, s_{2}, \ldots, s_{\ell}\right)$ be consecutive vertices on a face, ordered in clockwise or counter-clockwise order.

For each $i \in[\ell-1]$ and $\Delta \in\{-1,0\}$, define the set $A_{i}^{\Delta}:=\{v \in$ $\left.V: d\left(v, s_{i}\right) \leq d\left(v, s_{i+1}\right)+\Delta\right\}$. Define the universe $X:=[\ell] \times\{-1,0\}$, and the family

$$
\mathcal{F}(S):=\left\{\left\{(i, \Delta): v \in A_{i}^{\Delta}\right\}: v \in V\right\} \subseteq 2^{X} .
$$

Then, the VC dimension of $\mathcal{F}(S)$ (on universe $X$ ) is at most 3.

To prove the theorem, we use the following auxiliary lemma involving drawings in the plane below, whose easy but tedious proof is deferred to the full version.

We say that two (non-self-intersecting) arcs $C_{1}$ and $C_{2}$ cross, when the following holds: there are two points $p$ and $q$ on both $C_{1}$ and $C_{2}$ (possibly $p=q$ ) and a simple curve $C$ between $p$ and $q$ (possibly the single point $p$ if $p=q$ ) satisfying the following: there exists a $\epsilon_{0}>0$ such that for all positive $\epsilon<\epsilon_{0}$, the boundary of the region $B_{\epsilon}(C)$ of all points within distance $\epsilon$ from a point on $C$ has exactly four intersection points with $C_{1}$ and $C_{2}$, and they can be arranged in clockwise order so that the first and third points are on $C_{1}$ but not $C_{2}$, and the second and fourth are on $C_{2}$ but not $C_{1}$.

Lemma 3.8. Consider a simple, closed curve drawn in the plane, with eight (not necessarily distinct) points $p_{1}, p_{2}, \ldots, p_{8}$ placed clockwise around the curve that satisfy $p_{1} \neq p_{2}, p_{3} \neq p_{4}, p_{5} \neq p_{6}$, and $p_{7} \neq$ $p_{8}$. Consider two points $q_{1}, q_{2}$ inside the curve. It is impossible to draw $\operatorname{arcs}\left(q_{1}, p_{1}\right),\left(q_{1}, p_{4}\right),\left(q_{1}, p_{5}\right),\left(q_{1}, p_{8}\right),\left(q_{2}, p_{2}\right),\left(q_{2}, p_{3}\right),\left(q_{2}, p_{6}\right),\left(q_{2}, p_{7}\right)$ such that:

(1) The arcs from $q_{1}$ do not pairwise cross, and the arcs from $q_{2}$ do not pairwise cross, and

(2) the arcs from $p_{1}$ and $p_{2}$ do not touch, the arcs from $p_{3}$ and $p_{4}$ do not touch, the arcs from $p_{5}$ and $p_{6}$ do not touch, and the arcs from $p_{7}$ and $p_{8}$ do not touch.
Armed with Lemma 3.8, we now prove our VC dimension bound of Theorem 3.7.

Proof. Before we prove the theorem, we first remark that our proof will not actually use the fact that $\Delta$ takes on the values $-1,0$. Indeed, it can be adapted to work for $\Delta \in M$ for any set of real numbers $M$. This observation is needed for a smooth transition to the weighted case. We argue by contradiction: suppose that the set $\mathcal{F}$ has VC dimension at least 4 . Then, there exists a set

$$
Y:=\left\{\left(i_{1}, \Delta_{1}\right),\left(i_{2}, \Delta_{2}\right),\left(i_{3}, \Delta_{3}\right),\left(i_{4}, \Delta_{4}\right)\right\}
$$

such that for each subset $Y^{\prime} \subseteq Y$, there exists vertex $v \in V$ such that for each $a \in[4]$,

$$
v \in A_{i_{a}}^{\Delta_{a}} \Longleftrightarrow\left(i_{a}, \Delta_{a}\right) \in Y^{\prime} .
$$

First, we argue that the values $i_{1}, i_{2}, i_{3}, i_{4}$ are all distinct. Suppose, otherwise, that $i_{1}=i_{2}$, and assume without loss of generality that $\Delta_{1} \leq \Delta_{2}$. Consider the set $Y^{\prime}=\left\{\left(i_{1}, \Delta_{1}\right)\right\}$; by assumption, there must be a vertex $v \in V$ satisfying $v \in A_{i_{1}}^{\Delta_{1}}$ and $v \notin A_{i_{2}}^{\Delta_{2}}=A_{i_{1}}^{\Delta_{2}}$. This means that

$d\left(v, s_{i_{1}}\right) \leq d\left(v, s_{i_{1}+1}\right)+\Delta_{1} \quad$ and $\quad d\left(v, s_{i_{1}}\right)>d\left(v, s_{i_{1}+1}\right)+\Delta_{2}$, but $\Delta_{1} \leq \Delta_{2}$, so this is impossible, a contradiction.

Therefore, we can assume that $i_{1}, i_{2}, i_{3}, i_{4}$ are distinct, so assume without loss of generality that $i_{1}<i_{2}<i_{3}<i_{4}$. Consider the sets

$$
Y_{1}^{\prime}:=\left\{\left(i_{1}, \Delta_{1}\right),\left(i_{3}, \Delta_{3}\right)\right\} \quad \text { and } \quad Y_{2}^{\prime}:=\left\{\left(i_{2}, \Delta_{2}\right),\left(i_{4}, \Delta_{4}\right)\right\} .
$$

By assumption, there must be a vertex $t_{1} \in V$ such that

$$
\begin{array}{ll}
d\left(t_{1}, s_{i_{1}}\right) \leq d\left(t_{1}, s_{i_{1}+1}\right)+\Delta_{1}, & d\left(t_{1}, s_{i_{2}}\right)>d\left(t_{1}, s_{i_{2}+1}\right)+\Delta_{2}, \\
d\left(t_{1}, s_{i_{3}}\right) \leq d\left(t_{1}, s_{i_{3}+1}\right)+\Delta_{3}, & d\left(t_{1}, s_{i_{4}}\right)>d\left(t_{1}, s_{i_{4}+1}\right)+\Delta_{4},
\end{array}
$$

and a vertex $t_{2} \in V$ such that

$$
\begin{array}{ll}
d\left(t_{2}, s_{i_{1}}\right)>d\left(t_{2}, s_{i_{1}+1}\right)+\Delta_{1}, & d\left(t_{2}, s_{i_{2}}\right) \leq d\left(t_{2}, s_{i_{2}+1}\right)+\Delta_{2}, \\
d\left(t_{2}, s_{i_{3}}\right)>d\left(t_{2}, s_{i_{3}+1}\right)+\Delta_{3}, & d\left(t_{2}, s_{i_{4}}\right) \leq d\left(t_{2}, s_{i_{4}+1}\right)+\Delta_{4} .
\end{array}
$$

Consider the shortest paths between the pairs $\left(t_{1}, s_{i_{1}}\right),\left(t_{1}, s_{i_{2}+1}\right)$ $\left(t_{1}, s_{i_{3}}\right),\left(t_{1}, s_{i_{4}+1}\right),\left(t_{2}, s_{i_{1}+1}\right),\left(t_{2}, s_{i_{2}}\right),\left(t_{2}, s_{i_{3}+1}\right),\left(t_{2}, s_{i_{4}}\right)$.

We can assume that the paths from $t_{1}$ do not cross in their planar embeddings, since if two paths cross at a vertex $v \in V$, then we can modify one of the paths to agree with the other path up until vertex $v$, while still keeping it a shortest path.

Assume without loss of generality that the path $S=\left(s_{1}, \ldots, s_{\ell}\right)$ is numbered in clockwise order around the outer face. Since $i_{1}<$ $i_{2}<i_{3}<i_{4}$, the points $s_{i_{1}}, s_{i_{1}+1}, s_{i_{2}}, s_{i_{2}+1}, s_{i_{3}}, s_{i_{3}+1}, s_{i_{4}}, s_{i_{4}+1}$ are also in clockwise order around the outer face. Moreover, $s_{i_{1}} \neq$ $s_{i_{1}+1}, s_{i_{2}} \neq s_{i_{2}+1}, s_{i_{3}} \neq s_{i_{3}+1}$, and $s_{i_{4}} \neq s_{i_{4}+1}$. Therefore, we can invoke Lemma 3.8 on $\left(s_{i_{1}}, s_{i_{1}+1}, s_{i_{2}}, s_{i_{2}+1}, s_{i_{3}}, s_{i_{3}+1}, s_{i_{4}}, s_{i_{4}+1}\right)$ with the shortest paths from $t_{1}, t_{2}$. Note that Condition 1 of Lemma 3.8 is true, so it must be Condition 2 that is false. In other words, one of the following cases must hold:

(1) The shortest paths between $\left(t_{1}, s_{i_{1}}\right)$ and $\left(t_{2}, s_{i_{1}+1}\right)$ intersect at some vertex $v_{1} \in V$.

(2) The shortest paths between $\left(t_{1}, s_{i_{2}+1}\right)$ and $\left(t_{2}, s_{i_{2}}\right)$ intersect at some vertex $v_{2} \in V$.

(3) The shortest paths between $\left(t_{1}, s_{i_{3}}\right)$ and $\left(t_{2}, s_{i_{3}+1}\right)$ intersect at some vertex $v_{3} \in V$.

(4) The shortest paths between $\left(t_{1}, s_{i_{4}+1}\right)$ and $\left(t_{2}, s_{i_{4}}\right)$ intersect at some vertex $v_{4} \in V$. 
All four cases are similar, but due to space constraints, we only present Case 1 and defer the other cases to the full vesrion. Assuming Case 1 , define $P_{1,1}$ and $P_{2,2}$ to be the shortest paths between $\left(t_{1}, s_{i_{1}}\right)$ and $\left(t_{2}, s_{i_{1}+1}\right)$, respectively. Now, consider a path $P_{1,2}$ that travels from $t_{1}$ to $v_{1}$ along $P_{1,1}$, and then from $v_{1}$ to $s_{i_{1}+1}$ along $P_{2,2}$. Also, consider a path $P_{2,1}$ that travels from $t_{2}$ to $v_{1}$ along $P_{2,2}$, and then from $v_{1}$ to $s_{i_{1}}$ along $P_{1,1}$. For a path $P$, let len $(P)$ be the number of edges on the path $P$. We have

$$
\begin{aligned}
d\left(t_{1}, s_{i_{1}+1}\right)+d\left(t_{2}, s_{i_{1}}\right) & \leq \operatorname{len}\left(P_{1,2}\right)+\operatorname{len}\left(P_{2,1}\right) \\
& =\operatorname{len}\left(P_{1,1}\right)+\operatorname{len}\left(P_{2,2}\right) \\
& =d\left(t_{1}, s_{i_{1}}\right)+d\left(t_{2}, s_{i_{1}+1}\right) .
\end{aligned}
$$

However, summing up the inequality $d\left(t_{1}, s_{i_{1}}\right) \leq d\left(t_{1}, s_{i_{1}+1}\right)+\Delta_{1}$ from (2) and the inequality $d\left(t_{2}, s_{i_{1}+1}\right)+\Delta_{1}<d\left(t_{2}, s_{i_{1}}\right)$ from (3) gives $d\left(t_{1}, s_{i_{1}}\right)+d\left(t_{2}, s_{i_{1}+1}\right)+\Delta_{1}<d\left(t_{1}, s_{i_{1}+1}\right)+\Delta_{1}+d\left(t_{2}, s_{i_{1}}\right)$ which contradicts (5).

Thus, Sauer's lemma implies the following corollary, which concludes the proof of Theorem 3.2.

COROLlary 3.9. For the family $\mathcal{F}(S)$ defined in (1), we have $|\mathcal{F}(S)|=O\left(|S|^{3}\right)$.

The linear time computation of those tuples as well as the $(1+\epsilon)$ compression for weighted graphs are deferred to the full version.

\section{DISTRIBUTED DIAMETER IN UNWEIGHTED GRAPHS}

\subsection{Bounded Diameter Decomposition (BDD)}

For a graph $G$, we describe a recursive (balanced) partitioning procedure represented by a tree $\mathcal{T}$, whose vertices, denoted as bags, correspond to subgraphs in $G$. This recursive partitioning must satisfy two crucial properties. First, the diameter of each bag is kept being bounded by $O(D \log n)$ throughout all recursion levels which enables the computation of an $O(D \log n)$-path separator recursively. The second property provides a small overlap between all subgraphs in the same recursion level, by guaranteeing that each edge $e$ belongs to at most two subgraphs in each recursion level. This allows one to work on all subgraphs of the same level simultaneously with the same round complexity (up to constant factor) of working on a single subgraph.

Definition 4.1 (Bounded Diameter Decomposition). Let $G=(V, E)$ be a graph under some planar embedding and let $T$ be a BFS tree of $G$ of radius $D$ rooted at some node $r$. A bounded diameter decomposition $(B D D)$ of $G$ is a rooted tree $\mathcal{T}=\left(V_{\mathcal{T}}, E_{\mathcal{T}}\right)$ whose vertices $X \in V_{\mathcal{T}}$, called bags, are subsets of $V$ satisfying:

(1) The tree has depth at most $O(\log n)$.

(2) The root bag of $\mathcal{T}$ equals $V$.

(3) For each non-leaf bag $X$, its child bags $X_{1}, \ldots, X_{\ell}$ satisfy $\cup_{i} G\left[X_{i}\right]=G[X]$.

(4) Every leaf bag $X$ has size $O(D \log n)$.

(5) For each bag $X, G[X]$ is connected.

(6) For each bag $X, T[X]$ consists of at most $O(\log n)$ connected components.

(7) For each non-leaf bag $X$, consider the set $S_{X}$ of nodes in $X$ present in more than one child bag. There exist some $O(\log n)$ paths inside $T[X]$ whose disjoint union of vertices is exactly $S_{X}$. (This, together with the fact that $T$ has diameter $D$, implies that the size of this set is at most $O(D \log n)$.)

(8) There exists a mapping $O$ from the bags $X$ to connected open subsets of $\mathbb{R}^{2}$ such that:

(a) For each bag $X$, the planar embedding of the vertices and edges of $G[X]$ (which are points and simple curves in $\mathbb{R}^{2}$ ) are contained in $\overline{O(X)}$, the (topological) closure of $O(X)$.

(b) For each depth $d$, the subsets $O(X)$ over bags $X$ of depth $d$ are disjoint (in $\mathbb{R}^{2}$ ).

(9) For each bag $X$, there exists a closed curve in $\mathbb{R}^{2}$ through the nodes in $S_{X}$ that is disjoint from $O\left(X^{\prime}\right)$ for any child bag $X^{\prime}$.

THEOREM 4.2 (BOUNDED DIAMETER DECOMPOSITION FOR PLANAR GRAPHS). Let $G=(V, E)$ be an unweighted planar graph with diameter $D$. There is a distributed algorithm that computes the recursive partitioning of $G$ represented by a tree $\mathcal{T}$ of height $O(\log n)$ within $\widetilde{O}(D)$ rounds. In particular, every bag $X \in V_{\mathcal{T}}$ has a unique ID and every node knows the IDs of all the bags that contain it.

A few remarks. The set $S_{X}$ in property (7) fits the role of a separator in the graph $G[X]$. Also, properties (8) and (9) of the BDD may look cumbersome, but they necessary for the application of Theorem 3.2 in diameter computation. Indeed, they are the only two properties which involve the planarity of the graph. And since Theorem 3.2 assumes planarity, it makes sense that some aspects of planarity must be preserved in the BDD. Moreover, we use the topological notion of planarity (as opposed to combinatorial) because we need to track a fixed global embedding in our mind, so it is more geometrically intuitive to deal with regions in the plane.

Below, we present a few properties that are implied by the properties of the BDD that are sufficient for applications such as in Section 4.2 .

LEMmA 4.3. If $\mathcal{T}$ is a BDD for graph $G$, then the following additional properties hold:

3'. For each non-leaf bag $X$ and any two child bags $X_{1}$ and $X_{2}$, any path in $G[X]$ from a vertex in $X_{1}$ to a vertex in $X_{2}$ must intersect $S_{X}$.

6. For each bag $X, G[X]$ has diameter $O(D \log n)$.

7'. For each non-leaf bag $X,\left|S_{X}\right|=O(D \log n)$.

8'. For each depth d, every edge of $G$ is in at most 2 subgraphs $G[X]$.

\subsection{Distributed Computation of (Exact) Distance Labels}

In a seminal paper, Gavoille et al. [13] presented a distance labeling scheme with labels of size $O\left(r(n) \log ^{2} n\right)$ for the class of graphs with (recursive) $r(n)$-separators. Using $r(n)=O(\sqrt{n})$ yields the well known bound of $\widetilde{O}(\sqrt{n})$ distance labels. In our distance labels, we will use $r(n)=O(D \log n)$ for $n>D$. To make sure that an $O(D \log n)$-separator can be computed recursively, we use the BDD decomposition.

We first describe the labeling scheme by [13]. For the given graph $G$, the label $L_{G}(v)$ of each $v \in G$ consists of the following fields: (i) a list of distances $d_{G}(v, s)$ for every $s \in \operatorname{sep}(G)$, where $\operatorname{sep}(G)$ is the separator of $G$, (ii) the component ID of $v$ in $G \backslash \operatorname{sep}(G)$, and (iii) the (recursive) label $L_{G^{\prime}}(v)$ where $G^{\prime}$ is the component of $v$ in $G \backslash \operatorname{sep}(G)$. For sake of explanation, we denote the first two fields in the label 
by $\widehat{L}_{G}(v)$. Hence, a label of vertex $v$ consists of the concatenation of $k=O(\log n)$ sub-labels $L_{G}(v)=\widehat{L}_{G_{0}}(v) \circ \widehat{L}_{G_{1}}(v) \ldots \circ \widehat{L}_{G_{k}}(v)$ where $\widehat{L}_{G_{i}}(v)=\left\langle I D\left(G_{i+1}\right),\left\{\left(s, d_{G_{i}}(v, s)\right), s \in \operatorname{sep}\left(G_{i}\right)\right\}\right\rangle, G_{0}=G$ and $G_{i}$ is the component of $v$ in $G_{i-1} \backslash \operatorname{sep}\left(G_{i-1}\right)$ for every $i \in\{1, \ldots, k\}$. The decoding of the distance labels $L_{G}(u)$ and $L_{G}(v)$ is done by computing the minimum $u-v$ distance via the distances to the separator vertices in each level.

Our construction of the labels is also recursive, but requires a small adaptation from the scheme of [13]. Since our labels are based on $O(D \log n)$-size separator, the recursion is applied on the components of the BDD decomposition. Observe that in the recursive scheme of [13], all subgraphs in a given recursive level are vertex disjoint. This is because the child subgraphs of a graph $G^{\prime}$ are the connected components of $G^{\prime} \backslash \operatorname{sep}\left(G^{\prime}\right)$. This disjointness property is indeed crucial for the size bound of the final labels, as the label of a vertex $v$ contains the concatenation of the sublabels $\widehat{L}_{G^{\prime}}(v)$ for all the subgraphs $G^{\prime}$ in the recursion tree that contain $v$. In contrast, the level- $i$ subgraphs of the BDD decomposition are not necessarily vertex disjoint, as the vertices of the separators $S_{X}$ might be added to several subgraphs in order to keep the diameter small. To prevent a blow-up in the label size, we slightly modify the scheme of [13] as follows.

Initially, all vertices are marked. In every independent level of the BDD recursion with bag $X$, we are given a subgraph $G^{\prime}:=G[X]$ and assume first that $|X|=\Omega(D \log n)$. By property (7'), $S_{X}=$ $O(D \log n)$. The algorithm computes a BFS tree from each $s \in S_{X}$ in $G^{\prime}$. For the child bags $X_{1}, \ldots, X_{i}$ of $X$, define $G_{i}^{\prime}:=G^{\prime}\left[X_{i}\right]$. The label $L_{G}(v)$ of each marked vertex $v \in G^{\prime}$ is appended with the sublabel $\widehat{L}_{G^{\prime}}(v)$ which consists of (i) list of distances in $G^{\prime}$ from $v$ to each $s \in S_{X}$, and (ii) the ID of the subgraph $G_{j}^{\prime}$ to which $v$ belongs. Finally, all vertices in $S_{X}$ are unmarked. If $\left|G^{\prime}\right|=O(D \log n)$ (leaf bag in the decomposition), we compute a BFS tree from each $v \in G^{\prime}$, and append the label of each marked vertex in $G^{\prime}$ with the list of distances to every $u \in G^{\prime}$. This completes the description of the algorithm. The complete analysis is deferred to the full version.

\subsection{The Distributed Diameter Algorithm}

The diameter is computed on the BDD tree $\mathcal{T}$ from the leaf bags up to the root. The invariant for phase $i$ is that every node in every bag $X$ in level $D(\mathcal{T})-i+1$ knows $d(X)=\max _{u, v \in X} d_{G}(u, v)$. Note that $d(X)$ might be smaller than the diameter of $G[X]$, since it is based on the distances in $G$ rather than in $G[X]$. By keeping this invariant after each step, we get that after $D(\mathcal{T})=O(\log n)$ phases, the root vertex $r$ knows $D=d(V)$.

For the leaf bag $X$, simply assign a leader to collect the distance labels $L_{G}(v)$ of all vertices $v \in X$, locally compute $d(X)$, and then broadcast it to other nodes in the bag. Assume that the invariant holds up to phase $i$ for all bags in level $\ell_{i}=D(\mathcal{T})-i+1$, and we now describe phase $i+1$. Let $X$ be a bag in level $\ell_{i}-1$ and let $X_{1}, \ldots, X_{k}$ be its children bags in level $\ell_{i}+1$. By the invariant, every node in each child bag $X_{j}$ knows $d\left(X_{j}\right)$.

Let $u, v \in X$ be the pair of largest $G$-distance in $X$, i.e., $d(X)=$ $d_{G}(u, v)$. There are two options: (i) $u, v \subseteq X_{j}$ for some $j \in\{1, \ldots, k\}$, or (ii) $u \in X_{j}$ and $v \in X_{j^{\prime}}$ for some $j \neq j^{\prime} \in[k]$. Case (i) can be easily handled since nodes in $X_{j}$ know $d\left(X_{j}\right)$, and the maximum $d\left(X_{j}\right)$ value over all $j \in[k]$ can be computed along a BFS spanning tree in $G[X]$ of depth $O(D \log n)$, guaranteed by property (6'). Thus, the nodes in $X$ can compute $\max _{j} d\left(X_{j}\right)$.

Before we handle case (ii), let us define $G^{+}$to be all vertices and edges whose planar embedding is on or inside the closed curve guaranteed by property (9) of BDDs. Similarly, define $G^{-}$to be all vertices and edges whose planar embedding is on or outside the closed curve. Define $X^{+}$and $X^{-}$as the vertex sets of $G^{+}$and $G^{-}$, respectively. Observe that by property (9), every child $X_{j}$ satisfies $X_{j} \subseteq X^{+}$or $X_{j} \subseteq X^{-}$, but not both. For a vertex $v \in X$, define $G^{ \pm}(v)$ as the graph $G^{+}$or $G^{-}$that contains $v$. If both do (e.g., when $\left.v \in S_{X}\right)$, then choose one arbitrarily. Define $X^{ \pm}(v)$ as the vertex set of $G^{ \pm}(v)$. Finally, define $G^{\prime}:=G[X]$ and $G_{j}^{\prime}:=G\left[X_{j}\right]$ as before.

By property (3') of BDDs, any $u-v$ path in case (ii) must pass through a vertex in $S_{X}$. In particular, the shortest $u-v$ path must travel inside $X^{ \pm}(u)$ until reaching some node $s \in S_{X}$, then take the shortest $s-t$ path in $G$ to some $t \in S_{X}$ (possibly $t=s$ ), and finally travel inside $X^{ \pm}(v)$ to $v$. Therefore, it suffices to compute

$$
\max _{u \in X_{j}, v \in X_{j^{\prime}}, j \neq j^{\prime}} \min _{s, t \in S_{X}}\left(d_{G^{ \pm}(u)}(u, s)+d_{G}(s, t)+d_{G^{ \pm}(v)}(t, v)\right) .
$$

The value $d_{G}(s, t)$ in the min expression in (5) can be computed using the distance labels in $G$ : every node in $S_{X}$ can simply broadcast its distance label to all nodes in $X$.

For the other values $d_{G^{ \pm}(u)}(u, s)$ and $d_{G^{ \pm}(v)}(t, v)$, we compute distance in $G^{+}$and $G^{-}$so that every vertex $v \in G^{\prime}$ knows its $S_{X^{-}}$ distance tuple in $G^{ \pm}(v)$. Note that distances in $G$ will not work, since we want to apply Theorem 3.2 to the graphs $G^{+}$and $G^{-}$(which each have $S_{X}$ lying on a single face), and the theorem requires distances in $G^{+}$and $G^{-}$. To accomplish this for $G^{-}$, for each child bag $X_{j}$, first compute distance labels in $X_{j},{ }^{6}$ and have every pair of nodes $u, v \in S_{X} \cap X_{j}$ learn their distance in $G_{j}$. Then, consider an auxiliary graph $H$ on vertices $S_{X}$ where vertices $u, v \in S_{X}$ have an edge of length $\min _{G_{j}^{\prime} \subseteq G^{-}: u, v \in X_{j}} d_{G_{j}^{\prime}}(u, v$ ) (possibly $\infty$ ). Broadcast this graph to all nodes in $G^{-}$, so that each node $u \in X_{j} \subseteq X^{-}$ can compute, for each $t \in S_{X}, \min _{s \in S_{X} \cap X_{j}}\left(d_{G_{j}}(u, s)+d_{H}(s, t)\right)$. By property (3'), for every $u \in X^{-}$and $t \in S_{X}$, the (edges of the) shortest $u-t$ path can be broken into subpaths with endpoints in $S_{X}$ and which stay entirely inside some $G_{j}^{\prime}$, and the distance between these endpoints is correctly computed. Therefore, this correctly computes distances in $G^{-}$. We can then repeat the same argument for $G^{+}$.

Therefore, from now on, we assume that every vertex $v \in G^{\prime}$ has computed its $S_{X}$-distance tuple in $G^{ \pm}(v)$. Let us assume that all component IDs are represented by $B=O(\log n)$ bits. We then repeat the following process for each $b \in[B]$ : Let $\mathcal{B}_{b, 0}$ be all components $G_{j}^{\prime}$ whose $b^{\prime}$ th bit in their component ID is 0 , and let $\mathcal{B}_{b, 1}$ be those whose $b$ 'th bit in their component ID is 1 . Then, every vertex sends its distance tuple along the rooted spanning tree, together with the $b$ 'th bit of its component ID, so that the root learns the set of distinct

\footnotetext{
${ }^{6}$ The structure of the distance labels in Section 4.2 make computing these easy: simply read off the suffix of the distance label $L_{G}(v)=\widehat{L}_{G_{0}}(v) \circ \widehat{L}_{G_{1}}(v) \ldots \circ \widehat{L}_{G_{k}}(v)$ beginning at $\widehat{L}_{G_{j}^{\prime}}(v)$. Alternatively, if the reader wants to black-box Section 4.2, then the distance labels can be computed from scratch in each $G_{j}$. By property (8'), every edge is in at most two $G_{j}$ at this level, so the computations over different $G_{j}$ can be done simultaneously in $\widetilde{O}(D)$ rounds.

${ }^{7}$ Since there is only one child bag $X_{j}$ inside $X^{+}$, this case can be made even easier.
} 
(distance tuple, $b^{\prime}$ th bit of component ID) pairs. By Theorem 3.2, there are at most $\widetilde{O}\left(D^{4}\right)$ many distinct tuples, so this can be done efficiently, whose analysis is deferred to the Round Complexity section. Once the root of the spanning tree receives all these pairs, it computes

$$
\max _{\substack{u \in G_{j}^{\prime} \in \mathcal{B}_{b, 0} \\ v \in G_{j^{\prime}}^{\prime} \in B_{b, 1}}} \min _{s, t \in S_{X}}\left(d_{G^{\prime \pm}(u)}(u, s)+d_{G}(s, t)+d_{G^{\prime \pm}(v)}(t, v)\right),
$$

by trying all pairs of a $(\cdot, 0)$ tuple and a $(\cdot, 1)$ tuple. Finally, the two components $G_{j}, G_{j^{\prime}}$ achieving the max in Eq. (5) must have their components IDs differ in some bit position $b$, which means the root of the spanning tree correctly computes Eq. (5) for that value of $b$. So by trying all $b \in[B]$, one of them will succeed.

The complete analysis is in the full version. Finally, the computation of exact labels in weighted graphs, SSSP tree and $(1+\epsilon)$ approximation for weighted graphs are also deferred to the full version.

\section{REFERENCES}

[2] Amir Abboud, Keren Censor-Hillel, and Seri Khoury. 2016. Near-linear lower bounds for distributed distance computations, even in sparse networks. In International Symposium on Distributed Computing. Springer, 29-42.

[3] Amir Abboud, Pawel Gawrychowski, Shay Mozes, and Oren Weimann. 2018 Near-optimal compression for the planar graph metric. In Proceedings of the Twenty-Ninth Annual ACM-SIAM Symposium on Discrete Algorithms. SIAM, 530549.

[4] Sergio Cabello. 2017. Subquadratic algorithms for the diameter and the sum of pairwise distances in planar graphs. In Proceedings of the Twenty-Eighth Annual ACM-SIAM Symposium on Discrete Algorithms. SIAM, 2143-2152.

[6] Timothy M Chan and Dimitrios Skrepetos. 2017. Faster approximate diameter and distance oracles in planar graphs. In LIPIcs-Leibniz International Proceedings in Informatics, Vol. 87. Schloss Dagstuhl-Leibniz-Zentrum fuer Informatik.

[7] Panagiotis Charalampopoulos, Shay Mozes, and Benjamin Tebeka. 2019. Exact Distance Oracles for Planar Graphs with Failing Vertices. SODA (2019).

[8] Vincent Cohen-Addad, Søren Dahlgaard, and Christian Wulff-Nilsen. 2017. Fast and compact exact distance oracle for planar graphs. In Foundations of Computer Science (FOCS), 2017 IEEE 58th Annual Symposium on. IEEE, 962-973.

[9] Michael Elkin. 2017. Distributed exact shortest paths in sublinear time. In Proceedings of the 49th Annual ACM SIGACT Symposium on Theory of Computing. ACM, 757-770.

[10] Greg N Federickson. 1987. Fast algorithms for shortest paths in planar graphs, with applications. SIAM 7. Comput. 16, 6 (1987), 1004-1022.

[11] J.A. Garay, S. Kutten, and D. Peleg. 1993. A sub-linear time distributed algorithm for minimum-weight spanning trees. In Proc. of the Symp. on Found. of Comp. Sci. (FOCS).

[12] Cyril Gavoille, David Peleg, Stéphane Pérennes, and Ran Raz. 2001. Distance labeling in graphs. In Proceedings of the twelfth annual ACM-SIAM symposium on Discrete algorithms. Society for Industrial and Applied Mathematics, 210-219.

[13] Cyril Gavoille, David Peleg, Stéphane Pérennes, and Ran Raz. 2004. Distance labeling in graphs. fournal of Algorithms 53, 1 (2004), 85-112.

[14] Pawel Gawrychowski, Haim Kaplan, Shay Mozes, Micha Sharir, and Oren Weimann. 2018. Voronoi diagrams on planar graphs, and computing the diameter in deterministic $O\left(n^{5 / 3}\right)$ time. In Proceedings of the Twenty-Ninth Annual ACM-SIAM Symposium on Discrete Algorithms. SIAM, 495-514.

[15] Pawel Gawrychowski, Shay Mozes, Oren Weimann, and Christian Wulff-Nilsen 2018. Better tradeoffs for exact distance oracles in planar graphs. In Proceedings of the Twenty-Ninth Annual ACM-SIAM Symposium on Discrete Algorithms. SIAM, 515-529.

[16] Mohsen Ghaffari. 2015. Near-optimal scheduling of distributed algorithms. In Proceedings of the 2015 ACM Symposium on Principles of Distributed Computing. ACM, 3-12.

[17] Mohsen Ghaffari and Bernhard Haeupler. 2016. Distributed algorithms for planar networks I: Planar embedding. In the Proc. of the Int'l Symp. on Princ. of Dist. Comp. (PODC). 29-38.

[18] Mohsen Ghaffari and Bernhard Haeupler. 2016. Distributed algorithms for planar networks II: Low-congestion shortcuts, mst, and min-cut. In Proc. of ACM-SIAM Symp. on Disc. Alg. (SODA). 202-219.

[19] Mohsen Ghaffari and Jason Li. 2018. Improved distributed algorithms for exact shortest paths. In Proceedings of the 50th Annual ACM SIGACT Symposium on Theory of Computing. ACM, 431-444.

[20] Mohsen Ghaffari and Merav Parter. 2017. Near-Optimal Distributed DFS in Planar Graphs. In 31st International Symposium on Distributed Computing, DISC 2017, October 16-20, 2017, Vienna, Austria. 21:1-21:16.

[22] Bernhard Haeupler, D. Ellis Hershkowitz, and David Wajc. 2018. Round- and Message-Optimal Distributed Graph Algorithms. In Proceedings of the 2018 ACM Symposium on Principles of Distributed Computing, PODC 2018, Egham, United Kingdom, fuly 23-27, 2018. 119-128.

[23] Bernhard Haeupler, Taisuke Izumi, and Goran Zuzic. 2016. Low-congestion shortcuts without embedding. In Proceedings of the 2016 ACM Symposium on Principles of Distributed Computing. ACM, 451-460.

[24] Bernhard Haeupler, Taisuke Izumi, and Goran Zuzic. 2016. Near-Optimal LowCongestion Shortcuts on Bounded Parameter Graphs. In International Symposium on Distributed Computing. Springer, 158-172.

[25] Bernhard Haeupler and Jason Li. 2018. Faster Distributed Shortest Path Approximations via Shortcuts. In 32nd International Symposium on Distributed Computing, DISC 2018, New Orleans, LA, USA, October 15-19, 2018. 33:1-33:14

[26] Bernhard Haeupler, Jason Li, and Goran Zuzic. 2018. Minor Excluded Network Families Admit Fast Distributed Algorithms. In Proceedings of the 2018 ACM Symposium on Principles of Distributed Computing, PODC 2018, Egham, United Kingdom, fuly 23-27, 2018. 465-474.

[27] Chien-Chung Huang, Danupon Nanongkai, and Thatchaphol Saranurak. 2017. Distributed Exact Weighted All-Pairs Shortest Paths in Õ ( $\left.n^{\wedge}\{5 / 4\}\right)$ Rounds. In Foundations of Computer Science (FOCS), 2017 IEEE 58th Annual Symposium on. IEEE, $168-179$.

[28] Philip N Klein. 2005. Multiple-source shortest paths in planar graphs. In Proceedings of the sixteenth annual ACM-SIAM symposium on Discrete algorithms. Society for Industrial and Applied Mathematics, 146-155.

[29] Sebastian Krinninger and Danupon Nanongkai. 2017. A Faster Distributed SingleSource Shortest Paths Algorithm. arXiv preprint arXiv:1711.01364 (2017).

[30] Shay Kutten and David Peleg. 1995. Fast Distributed Construction of Kdominating Sets and Applications. In the Proc. of the Int'l Symp. on Princ. of Dist. Comp. (PODC). 238-251.

[31] Jason Li. 2018. Distributed Treewidth Computation. arXiv preprint arXiv:1805.10708 (2018)

[32] Richard J Lipton and Robert Endre Tarjan. 1979. A separator theorem for planar graphs. SIAM J. Appl. Math. 36, 2 (1979), 177-189.

[33] David Peleg. 2000. Distributed Computing: A Locality-sensitive Approach. Society for Industrial and Applied Mathematics, Philadelphia, PA, USA

[34] David Peleg, Liam Roditty, and Elad Tal. 2012. Distributed algorithms for network diameter and girth. In International Colloquium on Automata, Languages, and Programming. Springer, 660-672.

[35] Norbert Sauer. 1972. On the density of families of sets. Fournal of Combinatorial Theory, Series A 13, 1 (1972), 145-147.

[37] Mikkel Thorup. 2004. Compact oracles for reachability and approximate distances in planar digraphs. Fournal of the ACM ( ( ACM) 51, 6 (2004), 993-1024.

[38] Oren Weimann and Raphael Yuster. 2016. Approximating the diameter of planar graphs in near linear time. ACM Transactions on Algorithms (TALG) 12, 1 (2016), 12.

[39] Christian Wulff-Nilsen. 2008. Wiener index, diameter, and stretch factor of a weighted planar graph in subquadratic time. 